\title{
Characteristics of Social Relationships in a Group of Captive Asian Elephants (Elephas maximus) in the Elephant Village in Thailand
}

\author{
Saki Yasui ${ }^{1, *}$, and Gen'ichi Idani ${ }^{2}$ \\ ${ }^{1}$ Kyoto City Zoo \\ ${ }^{2}$ Wildlife Research Center, Kyoto University \\ *Corresponding author (Email: s2a1k9i@gmail.com)
}

Citation - Yasui, S., \& Idani, G. (2022). Characteristics of social relationships in a group of captive Asian elephants (Elephas maximus) in the elephant village in Thailand. Animal Behavior and Cognition, 9(1), 89-105. https://doi.org/10.26451/abc.09.01.08.2022

\begin{abstract}
Wild Asian elephants (Elephas maximus) form complex fission-fusion societies centered on matrilineal groups. In captivity, unrelated females remain in the same group and create social relationships. To better understand well-being in captivity, it is important to investigate the social relationships between females. However, to date, little information is available on this topic. The goal of this study was to clarify the social relationships between captive female Asian elephants using social network analysis. Our subjects were 13 captive Asian elephants at the Elephant Study Center in Surin Province, Thailand. We investigated variations in the frequencies of affiliative behaviors between dyads. Individuals that stayed in the group longer tended to play a more central role in terms of group member connections. We found that two individuals played an important role in strengthening connections, and that their removal influenced group cohesion. Our results revealed that individuals that stayed in the group long-term and that had existing relationships with others in the group tended to build better social connections, regardless of their age. In addition, the existence of some young individuals that show frequent affiliative behaviors may be important for bond strength and therefore, overall group cohesion.
\end{abstract}

Keywords - Asian elephants, Behavior, Social network analysis, Affiliative, Captivity

Asian elephants (Elephas maximus) are highly social animals. Recent studies on the social structure of Asian elephants have shown complex fission-fusion between groups (de Silva et al., 2011; Nandini et al., 2017). The "family group," which is centered on the mother and her offspring, forms the basic unit of elephant social structures (Sukumar, 1992). Females live in their natal groups throughout their lives, while males leave their natal group when they mature (Vidya \& Sukumar, 2005). Within family groups, elephants exhibit various social behaviors, such as allomothering and play (Joshi, 2009; Sukumar, 1992, 2011; Vidya, 2014). Through these behaviors, young elephants form social relationships and learn survival and social skills (Sukumar, 1992, 2011). Therefore, social relationships in elephant family groups are very important.

Unlike in the wild, many zoos keep unrelated female elephants in the same group, while the low breeding success in captivity suppresses the increase of related individuals in a group (Thitaram, 2012). Unrelated females in captive groups seem to show the same social behaviors as related females in wild family groups (Kurt \& Garaï, 2006; Rapaport \& Haight, 1987). However, few studies have investigated the social relationships between female Asian elephants (Garaï, 1992; Makecha et al., 2012). These studies show that social behavior varies between individuals. 
In the wild, it is challenging to collect detailed data on social interactions within Asian elephant family groups due to their large territories and the difficulty in observing them in their natural habitat. Captive elephants are considered suitable for such behavioral data collection because observations can be undertaken at close range, with focal sampling allowing the collection of enough behavioral data for analysis. Our subject group consisted of 13 captive elephants that lived with their mahouts (people who took care of the elephants and trained and worked with them) at a volunteer project (the Surin Project) in a Thai elephant village. Our subjects spent 3 to $6 \mathrm{hr}$ a day together, walking in the forest or staying at the enclosure included a pre-existing pond and trees. The members of our subject group were primarily unrelated females, chosen by humans, similar to individuals in most zoo-based elephant groups. It is rare for a captive elephant group to comprise more than 10 individuals (Rees, 2009). Even if there are limitations, interactions of 13 females belonging to various age classes in relatively natural environments would be worth investigating.

The objective of this study was to understand the relationship between captive female Asian elephants using social network analysis. Recently, social network analysis has been used to understand the social relationships and structures of various wild (Asian elephants: de Silva et al., 2011; African elephants: Wittemyer \& Getz, 2007; chimpanzees: Rushmore et al., 2013; dolphins: Lusseau, 2003), and captive species (brushtail possums: Corner et al., 2003; chimpanzees: Kanngiesser et al., 2011; rhesus macaques: Balasubramaniam et al., 2018). Understanding the interactions between female elephants may be useful for the management of captive Asian elephants. Such understanding leads to a better comprehension of the characteristics of affiliative networks and the factors that influence the group dynamics of unrelated female Asian elephants.

Many studies have shown that dominant individuals play central roles in grooming networks, both in the wild and in captivity (chimpanzees: Kanngiesser et al., 2011; Rushmore et al., 2013; primates: MacIntosh et al., 2012; Schino 2001). In wild African elephants, the eldest, largest, and most dominant females in each family group play an important role (McComb et al., 2001). This is also applicable to wild Asian elephant populations (Vidya \& Sukumar, 2005). In addition, large and dominant females tend to play a disciplinary role in captive groups of both Asian and African elephants (Freeman et al., 2004). Therefore, it is possible that older and dominant females play an important role in social relationships in captive groups as well. Affiliative behaviors such as grooming and proximity are used to evaluate social relationships in many species (Silk et al., 2013). Our first hypothesis is that older and dominant females play a central role in the affiliative network.

In captivity, elephants are sometimes transferred between facilities; it is therefore possible that younger individuals stay in a group longer than older individuals do. Individuals that stay in the group for a long time know the group members and have existing relationships. Therefore, our second hypothesis is that elephants that stay in the group for a long time are also important individuals in the affiliative network. To verify these hypotheses, we first built matrices of affiliative and aggressive behaviors and calculated the centrality measures (the main values featured in the networks). We could not define a clear dominance rank between subjects based on their behavior. We therefore used age as a rough guide for dominance, although we recognized that youngsters could be more dominant than elders in a wild population (de Silva et al., 2017). We hypothesized that older elephants or elephants that stayed in the group for a long time would have higher centrality measures. Next, we investigated whether the affiliative network had random or scalefree elements to investigate whether some individuals play more important roles than others in the network. Finally, we removed the central individuals and investigated the effect of their removal on network stability.

\section{Method}

\section{Ethical Statement}

The authors would like to thank the National Research Council of Thailand (NRCT) for their permission to conduct this study. 


\section{Study Site and Research Period}

We conducted our study at the Elephant Study Center in Ban Ta Klang Elephant Village, Surin Province, Thailand $\left(15^{\circ} 15^{\prime} 59.7^{\prime \prime} \mathrm{N}, 103^{\circ} 29^{\prime} 48.3^{\prime \prime} \mathrm{E}\right)$. Ban Ta Klang is a village of the Guay tribe, which is skilled in caring for, training, and working with elephants. Approximately 200 elephants were registered at the center during the study period. All of these elephants were taken care of by mahouts and lived with the mahouts' families. There are no wild elephants around this area now, although there used to be (Fernando \& Pastorini, 2011). Around 30-40 elephants worked in elephant shows or gave rides to tourists; some occasionally participated in parades or ceremonies. However, most of them were unoccupied throughout the day and spent a long time chained at shelters. Some elephants were kept by the same mahout families and were chained within touchable distance of each other and spent time together when they were unchained. Infants were usually unchained and stayed in fenced shelters with their chained mothers. Some elephants that had good relationships were sometimes chained in close proximity to each other at night in locations such as rice fields after harvest; here, chains were approximately $30 \mathrm{~m}$ in length so that they could move and interact with each other more freely than they could at shelters.

There are three seasons in this area of Thailand: summer (February-April), rainy (May-October), and winter (November-January) (Polthanee \& Promkhambut, 2014). We performed our research from December 2012 to March 2013.

\section{Subjects}

Our subjects were 13 captive-born female elephants from the Surin Project, ranging from 2-26 yrs old (Table 1). The Surin Project is a volunteer project run by a Thai non-profit organization (the Save Elephant Foundation) at Ban Ta Klang Elephant Village (Yasui \& Idani, 2017). This project aims to provide better living conditions for elephants in the village. Volunteers from all over the world join the project to learn about and experience the lives of the mahouts and elephants. Volunteers assist with the care of the elephants, including cleaning elephant shelters and planting or cutting grass and sugarcane. They also have the chance to go for a walk with the elephants at a distance, observe them in the enclosure from the platform, feed them, and wash them in the river. Participation fees from volunteers help finance the project.

Table 1

Members of the Study Group

\begin{tabular}{lccccc}
\hline Name (abb.) & Sex & Age (years) & Age class & Years on project & Focal time (h) \\
\hline Kaem sean (KS) & F & 26 & adult & 2.25 & 12.06 \\
Fah sai (FS) & F & 23 & adult & 3.42 & 11.12 \\
Mem (ME) & F & 20 & adult & 3.42 & 12.11 \\
Euang loaung (EL) & F & 18 & adult & 0.92 & 10.41 \\
Sai faa (SF) & F & 15 & subadult & 1.67 & 11.24 \\
Gem (GE) & F & 13 & subadult & 0.50 & 10.91 \\
Kanoon (KN) & F & 13 & subadult & 0.75 & 10.62 \\
Nong nung (NO) & F & 9 & juvenile & 1.92 & 11.83 \\
Nung ning (NU) & F & 9 & juvenile & 3.42 & 13.69 \\
Teng mo (TM) & F & 7 & juvenile & 1.58 & 12.55 \\
Ploy (PL) & F & 17 & adult & 0.17 & 6.26 \\
Khwan (KW; PL's daughter) & F & 2 & neonate & 0.17 & 6.26 \\
Kham koon (KK) & F & 5 & juvenile & - & - \\
\hline
\end{tabular}


The subjects were unrelated, except for one mother-calf dyad. Some of them were born in the village, while others were born in other areas in Thailand. They were reared by their mothers and separated from them at approximately 2-3 yrs of age so that they could be trained to work with the mahouts. All of them had been involved in street begging and had performed in shows before coming to the village and joining the project (Table A1). Three individuals, KS, ME, and PL, were parous and the others were nulliparous. The subjects saw many other elephants around the village every day, but they usually could not interact freely. The mahout leader chose which elephants would join the project from approximately 200 elephants that lived around the village. He primarily chose female elephants without any regular activities, then discussed rules and payment with the elephant's mahout. If the mahout agreed to the terms, the chosen elephant would join the group. Occasionally, he chose elephants that had a pre-existing positive relationship with another elephant in the group. The elephants in the project were all relatively young and active. It is possible that older elephants, such as those over 60 years old, were not chosen because they were not as active and strong as the young individuals, meaning they had a greater risk of serious injury, especially when they did not get along with other group members.

The elephants and mahouts followed a weekly schedule. In the mornings, at approximately 8:00, the mahouts took them to the area around the staff room of the Surin Project. The elephants were chained while their mahouts worked with volunteers to clean the shelters or plant grass or sugarcane. After that, the elephants were unchained and were able to walk around the village for approximately one hour (from 11:00 to 12:00). In the afternoon (approximately 14:00 to 16:00), after the mahouts and volunteers finished working, the elephants were chained in the project-owned enclosure in the village or went for a walk to the forest or the river and bathed. On Wednesdays, the elephants spent time without chain around the village for approximately $6 \mathrm{hr}$. After finishing the day's schedule, each mahout took his elephant home separately. The elephants were fed separately when they were chained around the staff room of the project, but were fed together during their walk and stay in the enclosure. The mahouts usually provided them with elephant grass or sugarcane, but they also ate various kinds of vegetation, such as bamboo, while walking in the forest. The elephants received veterinary care when veterinarians visited the village or were taken to veterinarians when needed.

\section{Behavioral Observations}

We observed our subjects when they spent time together in an enclosure or on walks for 3-6 hrs a day. We did not observe them when the mahouts or volunteers washed their elephants in the river or fed them separately. The elephants spent time walking in the forests and streets around the village, eating food, bathing in the river or ponds, and dusting with group members during the observation periods. The enclosure was made by fencing an area of approximately $3,000 \mathrm{~m}^{2}$; this included a pre-existing pond and trees. Mahouts, volunteers, and project staff usually accompanied the elephants during these activities. When the elephants were in the enclosure, only mahouts were present. The volunteers and staff always maintained a greater distance from the elephants than the mahouts did. In a few instances, the mahouts would attempt to stop interactions between elephants using vocal commands or physical contact, particularly during aggressive interactions. Therefore, it is possible that the observed frequencies of aggressive behaviors were lower than would have naturally occurred. The mahouts were not allowed to use hooks during project activities, which they would have normally used to control their elephants, as this was regarded as undesirable for elephant welfare and the project considered that female elephants could be controlled without hooks.

Of the 13 subjects, we targeted 12 elephants as the focal animals for this study (Table 1) because KK did not stay in the subject group for the entire observation period. KW was often tied to her mother, PL, with a rope (approximately $2 \mathrm{~m}$ ) around their necks. One author (Yasui) conducted all behavioral observations through focal animal sampling (Altmann, 1974) using an IC digital voice recorder (ICDUX523; Sony, Tokyo, Japan) and a video camera (HDR-550V; Sony, Tokyo, Japan). We randomly selected one focal animal for every walk and enclosure time and observed all subjects in all situations. During the long walks on Wednesdays, focal animals were switched between morning and afternoon. All social 
behaviors related to the focal animal were recorded, as were the names of the behavior actors and recipients. Elephant observational data are presented in Table 1. The total observation time was $129.1 \mathrm{~h}$, and each focal animal was observed 7-13 times (average $\pm S D: 9.5 \pm 1.7$ ), with an average duration of $1.19 \pm 0.61 \mathrm{hr}$. The distance between the target animal and the observer was 2-30 m. All elephants showed little interest in the observer during the study period, indicating that the observer had little influence on their behavior. We used the revised ethogram from our previous study to categorize the elephants' behaviors (Yasui \& Idani, 2017; Table 2).

Table 2

Ethogram of Social Behaviors (Yasui \& Idani, 2017 Revised)

\begin{tabular}{|c|c|}
\hline Behavior & Definition \\
\hline \multicolumn{2}{|l|}{ Move } \\
\hline Approach & $\begin{array}{l}\text { Move toward other individuals within the distance elephants can touch each other } \\
\text { (reach distance) }\end{array}$ \\
\hline Leave & Move away from the reach distance of other individuals \\
\hline Follow & Walk behind others keeping reach distance when they start walking, staying together \\
\hline \multicolumn{2}{|l|}{ Touch or smell } \\
\hline U-type touch & $\begin{array}{l}\text { Touch other elephant's body (lip, genitals, body, head, mouth, ear, leg, tail, trunk, } \\
\text { trunk tip) with U-shaped trunk }\end{array}$ \\
\hline S-type touch & $\begin{array}{l}\text { Touch other elephant's body (lip, genitals, body, head, mouth, ear, leg, tail, trunk, } \\
\text { trunk tip) with S-shaped trunk }\end{array}$ \\
\hline Touch with other body parts & $\begin{array}{l}\text { Touch other elephant's body with body parts other than the trunk tip, such as body, } \\
\text { tail, leg }\end{array}$ \\
\hline Trunk toward & Move trunk toward other elephants \\
\hline \multicolumn{2}{|l|}{ Aggressive } \\
\hline Head butt & Thump head against other elephant's head or body \\
\hline Trunk hit & Slap other elephant's head or body with trunk \\
\hline Kick & Kick other elephant's body with foreleg or hind leg \\
\hline Trunk/head over head & Put trunk or head to other elephant's head \\
\hline Trunk/head over back-aggressive & Put trunk or head to other elephant's back when the recipient is standing \\
\hline Push-aggressive & Push other elephant's head with raised head \\
\hline Push with tusk & Push other elephant's body with tusk \\
\hline \multicolumn{2}{|l|}{ Submissive } \\
\hline Presenting posterior & Walking backwards toward other elephants \\
\hline
\end{tabular}

\section{Data Analysis}

We defined all "U-type touches" (touching another elephant's body with a U-shaped trunk, Figure 1) as affiliative behaviors. Touching another elephant's body parts with the trunk tip has been recognized as an affiliative behavior in previous studies (Bonaparte-Saller \& Mench, 2018; Garaï, 1992; Makecha et al., 2012; Plotnik \& de Waal, 2014). In addition, touching the lips and genitals with U-shaped trunks was determined an affiliative behavior, as clarified in Yasui and Idani (2017). "Head butt," "trunk hit," "kick," "trunk/head over head," "trunk/head over back-aggressive," "push aggressive," and "push with tusk" (female Asian elephants have small tusks) were defined as aggressive behaviors as per previous studies (Bonaparte-Saller \& Mench, 2018; Makecha et al., 2012; Olson, 2004). To check the reliability of the observations, our research assistant watched 14 video clips (13.6 h in total) and counted the number of times affiliative and aggressive behaviors occurred between focal animals and other elephants in each video clip. To compare the data collected by the author (Yasui) and research assistant, we calculated the intraclass correlation coefficient, ICC $(3,1)$, for affiliative and aggressive behaviors, respectively, using the psych 
function in R (Revelle \& Revelle, 2015; Shrout \& Fleiss, 1979). The ICC $(3,1)$ for affiliative and aggressive behaviors were .96 and .85 , respectively, which were high enough to verify the author's observations.

Figure 1

An Example of U-Type Touching

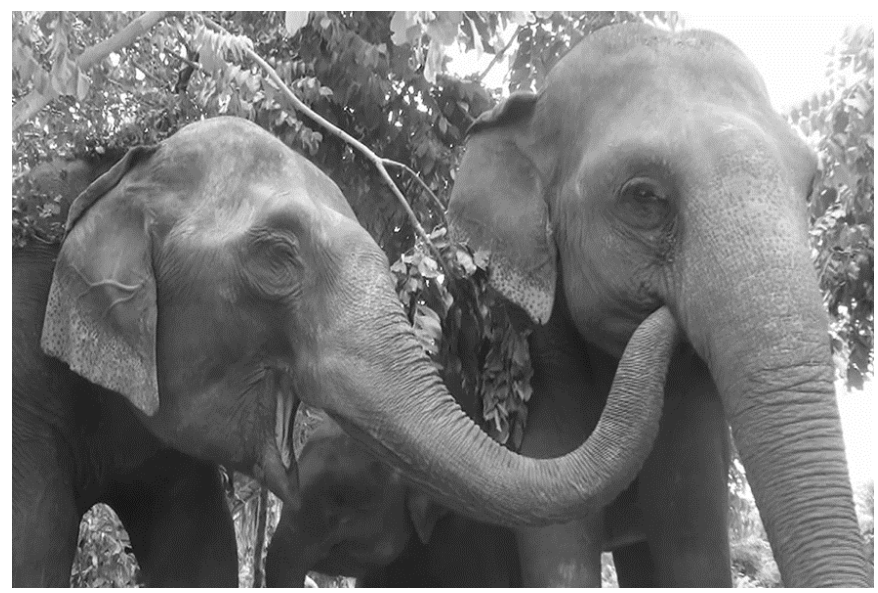

We calculated the frequency of affiliative and aggressive behaviors of all dyads as: $\left(O_{A B-A}+O_{A B-B}\right) /$ $\left(T_{A B}+T_{B A}\right)$, where $O_{A B-A}$ indicates the number of times $A$ showed affiliative/aggressive behaviors to $B$ when $A$ was the focal animal, and $O_{A B-B}$ indicates the number of times $A$ showed affiliative/aggressive behaviors to $B$ when $B$ was the focal animal. $T_{A B}$ indicates the time during which both $A$ and $B$ were in the study group, with $A$ as the focal animal, and $T_{B A}$ indicates the same measurement with $B$ as the focal animal.

First, we obtained weighted matrices of affiliative and aggressive behaviors in both the actor and recipient directions and checked the reciprocity of these behaviors using Dietz's R matrix correlation test in Socprog2.9 (Whitehead, 2009). To confirm "U-type touches" as an appropriate affiliative behavior, we checked the correlation between the proximity index and the frequencies of affiliative behaviors of dyads with Dietz's R matrix correlation test. The proximity indexes of all dyads were calculated as $\left(P_{A B}+P_{B A}\right) /$ $\left(T_{A B}+T_{B A}\right)$, where $P_{A B}$ indicates the time when $A$ and $B$ were within a touchable distance from each other, with $A$ as the focal animal, and $P_{B A}$ indicates the same measurement with $B$ as the focal animal. Touchable distance refers to the distance between two elephants, within which they can touch each other's body with their trunks, legs, or tails; this depends on the elephant's body size or position. We also built age, time, and familiarity matrices. In the age matrix, we used the age difference of each dyad. In the time matrix, we used the number of years in which each dyad stayed in the Surin Project. In the familiarity matrix, we set 1 if the dyad had stayed together before joining the project (KS-KN, FS-EL, and PL-KW) and 0 if the dyad had never stayed together before joining the project. To determine which parameters were related to affiliative and agonistic relationships, we investigated the correlation between an affiliative or aggressive matrix and age, time, and familiarity using Dietz's R matrix correlation test. We also checked the correlation between the proximity matrix and age, time, and familiarity. We used the data of KW because we could not proceed with the social network analysis with any missing data, although we recognized that the rope between KW and her mother, PL, influenced the interactions and proximity between the individuals of the group. However, the rope between KW and PL may have influenced the interactions data by a lesser extent compared to the proximity data. Therefore, we used interaction data for most analyses.

We calculated the network diameter (the longest path between shortest paths in the network), density (the fraction of observed paths in all possible paths), and global clustering coefficient (average degree that neighbors of an individual are also connected) of the affiliative and aggressive networks using Ucinet 6 (Borgatti et al., 2002; Newman, 2004a). We used hierarchical cluster analysis to test for subgroups using Socprog 2.9, and calculated the cophenetic correlation coefficients and maximum modularity. In this study, a 'subgroup' refers to a group with a smaller number of individuals than the whole subject group, 
led by hierarchical cluster analysis. This means that the members of each subgroup have more affiliative or agonistic relationships with each other than they do with others in the whole subject group, although all members of the subject group stay together. Values greater than 0.8 and 0.3 , respectively, indicate that the subgroupings are appropriate (Newman, 2004b, 2006). We used the group average method for clustering and built a matrix based on the results of hierarchical cluster analysis. Dyads that were clustered into the same subgroup were coded as 1 , while dyads that were clustered into different subgroups were coded as 0 . We then investigated the correlation of subgrouping with age, time, and familiarity using Dietz's R matrix correlation test.

For each individual, we calculated the degree (the number of relationships that each individual has with others), eigenvector centrality (the individual's influence in a network, reflecting the influence of its relationships and its neighbors' relationships), and betweenness centrality (how important each individual is to the connections of other individuals in the network) (Farine \& Whitehead, 2015). We investigated how these centrality measures were related to the age of each individual and the years in which each individual stayed in the Surin Project ("years in the project") using the double-semi-partitioning (DSP) procedure (Franks et al., 2021). We built a regression model by setting the centrality measures as response variables and age and years in the project as explanatory variables. We conducted the DSP with 10,000 permutations of the residuals using R scripts provided by Franks et al. (2021). We used eigenvector and betweenness centralities for the curve estimation test to investigate whether the affiliative network had characteristics of random or scale-free networks. When a network has scale-free properties, a few individuals play more central and important roles than others in the network. We used a linear function for the random network and a power function for the scale-free network. We checked Akaike's information criterion (AIC) for each function and used the propagation function to calculate and plot the confidence interval of the fitted nonlinear model (Spiess, 2018). Subsequently, we isolated some individuals with high centrality measures (central individuals) using the function called "influence.measures" in R (Fox \& Monette, 2002).

To investigate the influence of central individuals on the stability of an affiliative network, we simulated two conditions. In the targeted conditions, we removed the central individuals from the network (Bret et al., 2013). Individuals were removed under random conditions by random sampling using the "sample" function in R (Lusseau, 2003); the removal was repeated ten times (Kanngiesser et al., 2011; Manno, 2008). We calculated the diameter, fragmentation, and density of the network in each condition using Ucinet 6.0. All statistical analyses were performed using the R software (ver. 3.5.1; R Core Team, 2018).

\section{Results}

\section{Basic Characteristics of Affiliative and Aggressive Networks}

The affiliative and aggressive behaviors in dyads were reciprocal in both periods (Dietz's R matrix correlation: affiliative: $r=.75, p<.01$; aggressive: $r=.72, p<.01$ ). Therefore, we used the total number of times that affiliative or aggressive behaviors were observed in each dyad for subsequent analyses. We confirmed that the affiliative and proximity matrices were correlated (Dietz's R matrix correlation: $r=.83$, $p<.01)$.

We observed affiliative behaviors in 60 dyads; six dyads (ME-EL, EL-PL, SF-KN, SF-PL, SF-KW, and GE-KW) did not show affiliative behaviors. The average frequencies $( \pm S D)$ of affiliative behaviors between dyads were $0.99 \pm 2.03$ times/hr. The matrix of affiliative behaviors was moderately correlated with the familiarity matrix (Dietz's R matrix correlation: $r=.28, p=.01$ ). Age and time matrices were not correlated with the affiliative matrix (Dietz's R matrix correlation: age: $r=.10, p=.44$; time: $r=.19, p=$ .15). The proximity matrix was also correlated with the familiarity matrix and was not correlated with the age and time matrices (Dietz's R matrix correlation: familiarity: $r=.29, p=.02$; age: $r=.10, p=.45$; time: $r=.19, p=.16)$. The diameter, density, and global clustering coefficients of the affiliative network were 2 , 0.99 , and 1.44, respectively. We identified three groups containing two to four individuals, each; two individuals (KS and NU) did not belong to any subgroup (Figure 2, cophenetic correlation coefficient $=$ 
.97 , maximum modularity $=.54$ ). The result of subgrouping was not explained by age, time, or familiarity (Dietz's R matrix correlation: age: $r=-.11, p=.43$; time: $r=.24, p=.09$; familiarity: $r=-.07, p=.74$ ).

\section{Figure 2}

Weighted Network of Affiliative Interactions $(N=12)$

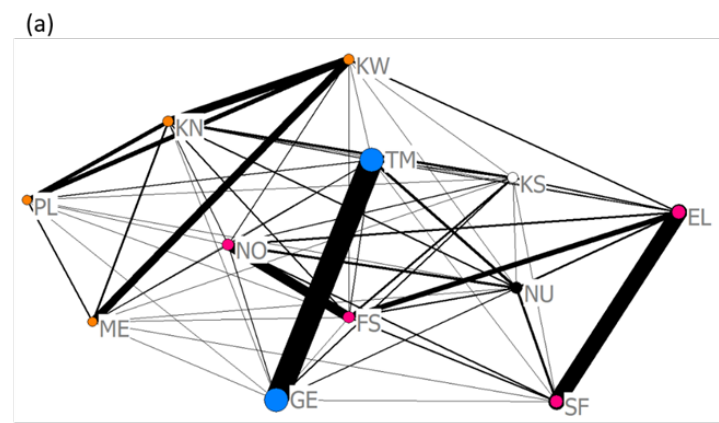

(b)

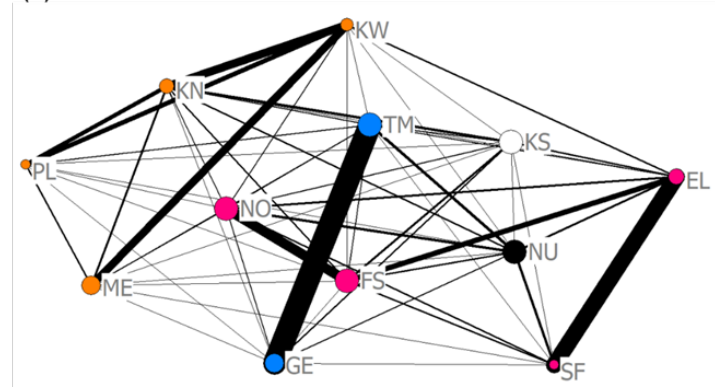

Note. Nodes represent individuals, and a larger node size indicates higher eigenvector centrality (a) and betweenness centrality (b). The width of the lines represents the frequency between the dyads. The same color indicates the same subgroup.

Only 6 out of 66 dyads (KS-FS, FS-SF, FS-KN, FS-GE, SF-NU, and NO-NU) showed aggressive behaviors. These six dyads differed in their entirety from the six dyads that did not show affiliative behaviors. The average frequencies of aggressive behaviors among these dyads were $0.02 \pm 0.08 \mathrm{times} / \mathrm{hr}$. The aggressive matrix was not correlated with any parameters (Dietz's R matrix correlation: age: $r=.04, p$ $=.84$; time: $r=.02, p=.78$; familiarity: $r=.26, p=.10$ ). The diameter, density, and global clustering coefficients of the affiliative network were $4,0.09$, and 0.00 , respectively. We identified two subgroups containing three individuals, each; six individuals did not belong to any subgroup (cophenetic correlation coefficient $=.93$, maximum modularity $=.60$ ). Subgrouping was not correlated with age, time, or familiarity (Dietz's R matrix correlation: age: $r=-.10, p=.42$; time: $r=.26, p=.07$; familiarity: $r=.18, p=.27$ ). Aggressive interactions in the subject group were very rare, and the network was not well connected. Therefore, we decided to use only affiliative behavior data for further analysis. In addition to the very low frequency of aggressive behavior, we did not observe any submissive behavior. Therefore, we could not define a clear dominance rank among the subjects.

\section{Centrality Measures of Affiliative Network}

The centrality measures for the affiliative behavior network are presented in Table 3. Betweenness centralities were positively correlated with years in the project (coefficient $\pm S E: 0.15 \pm 0.06, t=2.70, p=$ .030 ; Table A2), although they were not correlated with age (coefficient $\pm S E$ : $-0.01 \pm 0.01, t=-0.58, p=$ 
.59; Table A2). Degrees and eigenvector centralities were not significantly correlated with age or years in the project (coefficient $\pm S E$ : $-0.79--0.01 \pm 0.01-0.93, t=-1.04--0.36, p>.32$; Table A2).

Table 3

Affiliative Behavior Network Indices

\begin{tabular}{lccc}
\hline Name & Degree & Eigenvector centrality & Betweenness centrality \\
\hline KS & 5.01 & 0.10 & 0.76 \\
FS & 12.08 & 0.17 & 0.76 \\
ME & 7.25 & 0.07 & 0.52 \\
EL & 15.27 & 0.22 & 0.38 \\
SF & 11.91 & 0.19 & 0.11 \\
GE & 14.67 & 0.63 & 0.50 \\
KN & 11.04 & 0.11 & 0.35 \\
NO & 10.6 & 0.16 & 0.76 \\
NU & 6.98 & 0.15 & 0.76 \\
TM & 16.09 & 0.63 & 0.76 \\
PL & 6.6 & 0.08 & 0.11 \\
KW & 13.02 & 0.10 & 0.24 \\
\hline
\end{tabular}

To investigate whether the affiliative network was random or scale-free, we checked the cumulative frequencies of the eigenvector and betweenness centralities. The cumulative distribution of eigenvector centrality was fitted with a power function (Figure $3 \mathrm{a}$; power curve estimation test: $A I C=56.34, F_{1,11}=$ 54.58, $p<.01$; linear curve estimation test: $A I C=62.79, F(1,11)=22.33, p<.01)$. The cumulative distribution of betweenness centrality was also fitted with a power function (Figure $3 \mathrm{~b}$; power curve estimation test: $A I C=40.80, F(1,11)=205.26, p<.01$; linear curve estimation test: $A I C=40.93, F(1,11)$ $=168.1, p<.01)$. However, the estimated power model was $\mathrm{y}=13.360 \mathrm{x}^{1.0547}$, and the shape of the graph differed from the expected shape, showing scale-free characteristics. Regarding eigenvector centrality, the influence measures test showed that GE and TM presented higher values than did others.

\section{Influence of Central Individuals}

In the targeted conditions, we removed individuals with high eigenvector centralities, GE and TM. However, the diameter of the network did not change after removing any individual. Removal of TM increased the fragmentation of the affiliative network (Figure 4a; initial network: $f=0.045$; removal of TM: $f=0.055)$. Additionally, removal of KS, FS, NO, and NU increased the fragmentation of the network to the same level as that of TM ( $f=0.055$ for all four individuals). Removal of GE and random removal did not affect network fragmentation (Figure 4a; initial network: $f=0.04$; removal of GE: $f=0.045$; random condition: $f=0.043 \pm 0.008$ ). The density of the network decreased with the removal of TM and GE (Figure $4 \mathrm{~b}$; initial network: $d=0.99$; removal of TM: $d=0.89$; removal of GE: $d=0.91$ ). Random removal did not influence the density of the network (initial network: $d=0.99$; random condition: $d=0.96 \pm 0.07$ ). 
Figure 3

Cumulative Frequencies of Eigenvector Centrality (a) and Betweenness C entrality (b) about Affiliative Behaviors

(a)

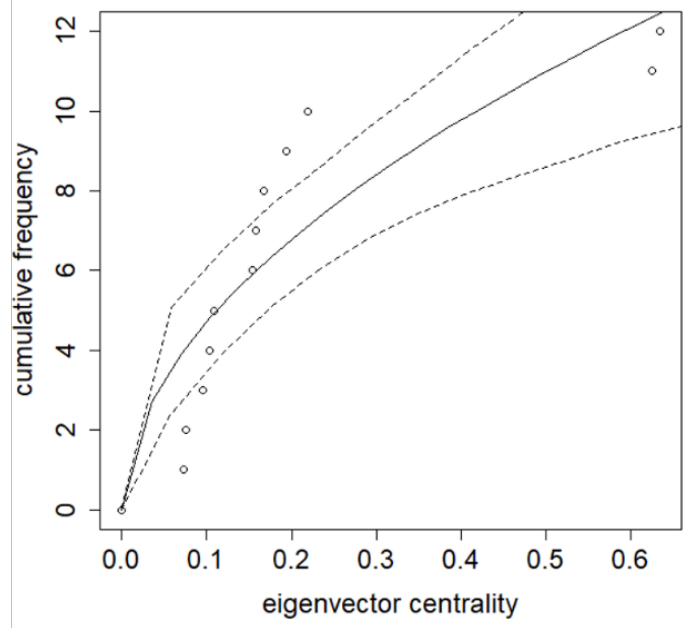

(b)

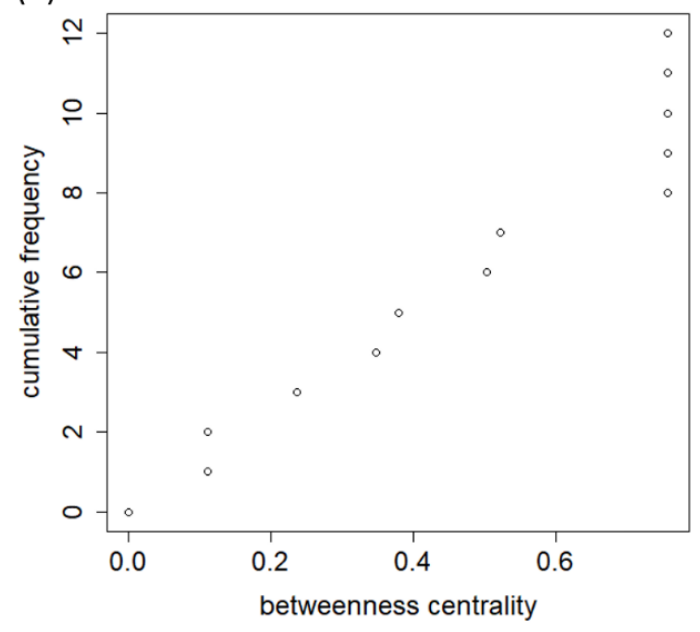

Note. The solid line shows the fitted power model, and dashed lines show the $95 \%$ confidence intervals.

\section{Figure 4}

The Changes of Fragmentation (a) and Density (b) of the Affiliative Network by Removal of the Central Individuals

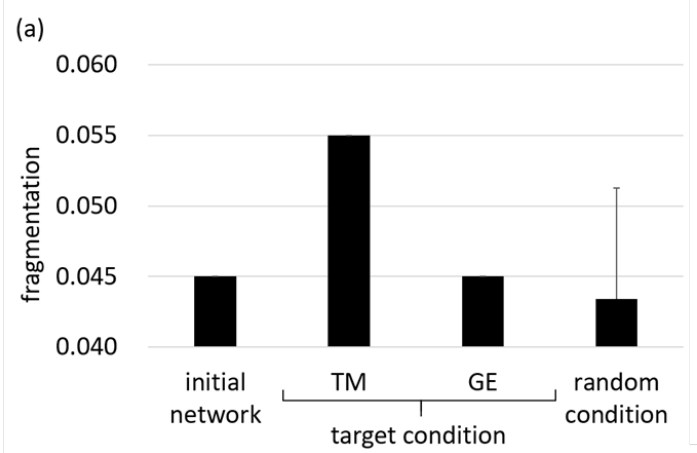

(b)

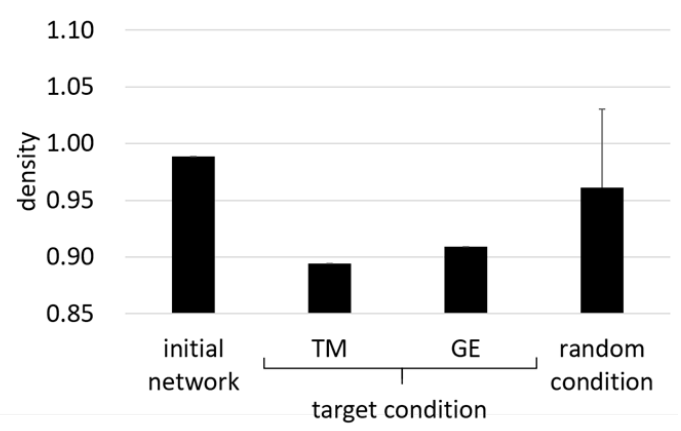

Note. The error bars show standard deviation 


\section{Discussion}

The affiliative network of our subject group was very dense; subjects frequently communicated in affiliative ways, while they seldom showed aggressive interactions. The dyads that had stayed together before joining the project tended to show higher affiliative behaviors than the other dyads. This result shows that familiarity is likely to be important for social relationships among female elephants. de Silva et al. (2017) reported that wild Asian elephants usually tend to avoid meeting and interacting with individuals from other social groups, which might lead to rare aggressive behaviors and an unclear dominance hierarchy. However, this does not mean that wild Asian elephants do not have a fission-fusion social structure. As de Silva and Wittemyer (2012) described, they show fission-fusion, but their social groups congregate together less frequently compared to the social groups of wild African savannah elephants. In zoos, a low frequency of aggressive behaviors has been observed compared to affiliative behaviors (Garaï 1992; Harvey et al., 2018; Makecha et al., 2012), although our subjects showed fewer aggressive behaviors than those reported by Makecha et al. (2012). In this study, aggressive interactions between the subjects may have been suppressed by the mahouts' interventions, although they usually could not stop the initiation of aggression. In addition, our subjects stayed together for only 3 to 6 hours a day and lived separately for the rest of the day, whereas female elephants in zoos are always together in limited spaces, and show a relatively clear dominance rank (Makecha et al., 2012). It therefore may not have been necessary for our subjects to establish a clear dominance hierarchy because they did not need to share food or space with other group members as zoo elephants do.

In the hierarchical cluster analysis, we found three subgroups with two to four individuals in the affiliative network. In addition, as shown in Figure 2, each individual showed frequent affiliative behaviors with few individuals. Studies of wild Asian elephants have shown average group sizes of 2.38 and 3.07 in Kabini, India, and Uda Walawe and Sri Lanka, respectively (de Silva \& Wittemyer, 2012; Nandini et al., 2018). Recent studies on the social structure of Asian elephants have shown that their group size is restricted, even if their "clan" size is large (de Silva et al., 2011; Nandini et al., 2017). Clan is the most inclusive and stable level of female social structure, and it includes three levels of social group at most (Nandini et al., 2018). The size of the subgroups in our study was similar to the group sizes of wild populations, although our subjects were unrelated, unlike wild populations. This might mean that, due to the nature of their sociality, female Asian elephants form strong affiliative relationships with a small number of individuals, regardless of relatedness. These results also indicate that it is possible for only a few individuals in a captive group to have close relationships, with some individuals, such as KS and NU, not forming relationships with herd mates at all. de Silva et al. (2017) reported that the observed frequency of solitary adult females is higher in wild Asian elephants than in wild African savannah elephants. This suggests that female Asian elephants do not always require close relationships or frequent interactions with other individuals. Our results may also suggest that it is important to give elephants enough space to avoid interactions with others if they choose to in captivity, although it is not considered desirable for them to be isolated for long periods.

The elephants that stayed in the project for a longer time tended to have higher betweenness centralities. However, age was not related to any centrality measure. This result partly supports our second hypothesis and suggests that, in captivity, existing relationships between group members could be more important for affiliative interactions than their age in terms of mediating between other members in the group.

The results of cumulative frequencies in eigenvector centralities showed that the affiliative network had scale-free properties, with some individuals playing a more important role in the network than others. Two individuals, GE and TM, were defined as central individuals based on the results of the influence measures test about eigenvector centrality. A network with scale-free properties has been found in several mammalian species (dolphins: Lusseau, 2003; ground squirrels: Manno, 2008; mandrills: Bret et al., 2013; northern myotis: Johnson et al., 2012). Our results support a previous study that suggested that scale-free and random networks could be adapted to small sample sizes (Kanngiesser et al., 2011). To the best of our knowledge, this is the first report to show evidence of scale-free properties in elephants. 
In addition, we conducted a simulation to investigate network stability by removing the central individuals. The removal of GE and TM did not affect the network diameter. This is because the affiliative network was very dense. However, the removal of GE and TM decreased the density. GE and TM showed affiliative behaviors very frequently, especially between the two of them. This may contribute to their role as central individuals in eigenvector centrality. The removal of TM fragmented the network, whereas removal of GE did not. TM showed affiliative behaviors with all other members in the group, which led to the fragmentation of the network upon TM's removal. We should note that the removal of the other four individuals that had the highest betweenness centrality also fragmented the network. Our results revealed that GE contributed to the group cohesion in terms of bond strength and TM in terms of bond strength and connection between members. Our results support previous studies on other species that showed that the interaction network was sensitive to the removal of central individuals (killer whales: Williams \& Lusseau, 2006; ground squirrels: Manno, 2008; chimpanzees: Kanngiesser et al., 2011). TM and GE were 7 and 13 years old, respectively, and they were smaller than the adults. TM did not show aggressive behavior during observation, while GE received aggressive behaviors only once (from FS) and never initiated them. It was difficult for us to judge them as the most dominant individuals; we could not confirm the dominance ranks among the subjects because of infrequent aggressive behavior and the lack of submissive behavior. There is a need to investigate the relationship between dominance and centrality measures, but our results show that factors other than age or dominance rank can be more important in affiliative networks in captive Asian elephants. Some studies on captive elephants have shown that subadults or juveniles interact in an affiliative manner more frequently than adults (Garaï, 1992; Harvey et al., 2018). Our results for subgrouping suggest that each individual needs only a few other individuals for close relationships. However, considering whole group cohesion in animals sharing a limited space in captivity, the existence of young individuals that frequently show affiliative interactions with other members, even if there is a difference in frequency, may be important. The removal of individuals in this study replicates the death or translocation of individuals in captivity. It is easy to guess that the loss of matrices in groups will induce great changes in social relationships between the other group members. Based on this study, the death or translocation of young females may cause great damage to group cohesion.

We recognize that our subjects are individuals of a small captive group of Asian elephants in a limited and not easily replicated situation. Our subject group was different from a group of wild elephants in terms of relatedness and the inclusion of human choice in forming the group. In captivity, elephants need to stay together in a limited space, regardless of whether they get along or not. This is likely to lead to behavioral differences between captive and wild elephant groups. Our subjects did not include elephants over 30 years of age. Elephants in their 20s are considered fully-grown adults (Arivazhagan \& Sukumar, 2008; Kurt \& Garaï, 2006); however, a lack of older and more experienced elephants may lead to changed behaviors. In addition, our subjects did not stay together full-time and always stayed with their mahouts during observations. These situations differed not only from those of wild elephants, but also from those of most zoo elephants. As previously mentioned, these factors may influence the frequency of aggressive behaviors along with dominance relationships, and the special characteristics of the project living conditions may have had a greater effect on our subjects. Given that our subject group differs from wild or captive zoo elephant groups, our results must only be considered to provide a general overview of female elephant social interactions, the details of which must be confirmed with further investigation. However, our subject group is considered to be more similar to groups of wild elephants than those of zoo elephants at some points. In our study group, new group member selections sometimes included elephants that had existing, positive relationships with elephants that were already a part of the subject group. This process placed more importance on the relationships made by the elephants themselves than is often possible in a zoo environment. The age composition of our subject group seemed to be more similar to that of wild elephant groups than that of captive zoo elephant groups; previous captive studies have shown little age variation in subject groups (Garaï, 1992; Makecha et al., 2012). In addition, the social relationships between female elephants have rarely been studied for groups of more than 10 individuals, even in captivity. Therefore, this study is important in terms of revealing social relationships using social network analysis, despite its limitations, as it considers relationships between 12 female elephants of various ages in a much 
more natural environment than is found in zoo exhibits. This study represents a new step in the process of investigating social relationships within groups.

Captive elephants live in various facilities such as zoos, sanctuaries, and elephant camps; the conditions for elephants in these facilities vary significantly. Therefore, studies on social relationships between elephants in various captive situations should be conducted in the future. Various factors such as group composition, the behavioral tendencies of key individuals, and origin seemed to affect the variability of social networks in chimpanzees (Cronin et al., 2014; Levé et al., 2016; van Leeuwen et al., 2013). Judging from their social complexity and high cognitive ability, elephants should have high social flexibility, similar to chimpanzees. Therefore, in elephants, conducting social network analyses in various populations, including wild ones, and comparing the results between populations or species in the future will help better understand elephant welfare in captivity as well as their sociality.

Conflict of Interest: The authors have no conflict of interest associated with the content of this article.

\section{Acknowledgements}

The authors would like to thank the National Research Council of Thailand (NRCT) for their permission to conduct this study. We appreciate our research collaborator, Dr. Wanchai Tunwattana, a project manager for the Elephant Kingdom Project, Zoological Parks Organization. We thank all the staff and mahouts of the Surin Project for their cooperation. We are grateful to Yumi Yamanashi, Tomoko Matsusaka, Sota Inoue, and Tamao Maeda for their help in analyzing our data and improving our manuscript. We would also like to thank Editage (www.editage.jp) for English language editing. This work was supported by a grant-in-aid from the Japan Society for the Promotion of Science (JSPS) fellows (grant number 245462), and the ITP-HOPE and AS-HOPE programs from JSPS.

\section{References}

Altmann, J. (1974). Observational study of behavior: Sampling methods. Behaviour, 49(3), 227-266.

Arivazhagan, C., \& Sukumar, R. (2008). Constructing age structures of Asian elephant populations: A comparison of two field methods of age estimation. Gajah, 29, 11-16.

Balasubramaniam, K., Beisner, B., Guan, J., Vandeleest, J., Fushing, H., Atwill, E., \& McCowan, B. (2018). Social network community structure and the contact-mediated sharing of commensal E. coli among captive rhesus macaques (Macaca mulatta). PeerJ, 6, e4271.

Bonaparte-Saller, M., \& Mench, J. A. (2018). Assessing the dyadic social relationships of female African (Loxodonta africana) and Asian (Elephas maximus) zoo elephants using proximity, tactile contact, and keeper surveys. Applied Animal Behaviour Science, 199, 45-51.

Borgatti, S. P., Everett, M. G., \& Freeman, L. C. (2002). Ucinet 6 for Windows: Software for Social Network Analysis. Analytic Technologies.

Bret, C., Sueur, C., Ngoubangoye, B., Verrier, D., Deneubourg, J. L., \& Petit, O. (2013). Social structure of a semifree ranging group of mandrills (Mandrillus sphinx): A social network analysis. PloS One, 8(12), e83015.

Corner, L. A. L., Pfeiffer, D. U., \& Morris, R. S. (2003). Social-network analysis of Mycobacterium bovis transmission among captive brushtail possums (Trichosurus vulpecula). Preventive Veterinary Medicine, 59(3), 147-167.

Cronin, K. A., Van Leeuwen, E. J., Vreeman, V., \& Haun, D. B. (2014). Population-level variability in the social climates of four chimpanzee societies. Evolution and Human Behavior, 35(5), 389-396.

de Silva, S., Ranjeewa, A. D., \& Kryazhimskiy, S. (2011). The dynamics of social networks among female Asian elephants. BMC Ecology, 11(1), 17.

de Silva, S., Schmid, V., \& Wittemyer, G. (2017). Fission-fusion processes weaken dominance networks of female Asian elephants in a productive habitat. Behavioral Ecology, 28(1), 243-252.

de Silva, S., \& Wittemyer, G. (2012). A comparison of social organization in Asian elephants and African savannah elephants. International Journal of Primatology, 33(5), 1125-1141.

Farine, D. R., \& Whitehead, H. (2015). Constructing, conducting and interpreting animal social network analysis. Journal of Animal Ecology, 84(5), 1144-1163.

Fernando, P., \& Pastorini, J. (2011). Range-wide status of Asian elephants. Gajah, 35, 15-20. 
Fox, J., \& Monette, G. (2002). An R and S-Plus companion to applied regression. Sage Publications.

Franks, D. W., Weiss, M. N., Silk, M. J., Perryman, R. J., \& Croft, D. P. (2021). Calculating effect sizes in animal social network analysis. Methods in Ecology and Evolution, 12(1), 33-41.

Freeman, E. W., Weiss, E., \& Brown, J. L. (2004). Examination of the interrelationships of behavior, dominance status, and ovarian activity in captive Asian and African elephants. Zoo Biology, 23(5), 431-448.

Garaï, M. E. (1992). Special relationships between female Asian elephants (Elephas maximus) in zoological gardens. Ethology, 90(3), 187-205.

Harvey, N. D., Daly, C., Clark, N., Ransford, E., Wallace, S., \& Yon, L. (2018). Social interactions in two groups of zoo-housed adult female Asian elephants (Elephas maximus) that differ in relatedness. Animals, 8(8), 132.

Johnson, J. B., Ford, W. M., \& Edwards, J. W. (2012). Roost networks of northern myotis (Myotis septentrionalis) in a managed landscape. Forest Ecology and Management, 266, 223-231.

Joshi, R. (2009). Asian elephant's (Elephas maximus) behaviour in the Rajaji National Park, North-West India: Eight years with Asian elephants. Nature and Science, 7(1), 49-77.

Kanngiesser, P., Sueur, C., Riedl, K., Grossmann, J., \& Call, J. (2011). Grooming network cohesion and the role of individuals in a captive chimpanzee group. American Journal of Primatology, 73(8), 758-767.

Kurt, F., \& Garaï, M. E. (2006). The Asian elephant in captivity: A field study. Cambridge University Press.

Levé, M., Sueur, C., Petit, O., Matsuzawa, T., \& Hirata, S. (2016). Social grooming network in captive chimpanzees: Does the wild or captive origin of group members affect sociality? Primates, 57(1), 73-82.

Lusseau, D. (2003). The emergent properties of a dolphin social network. Proceedings of the Royal Society of London. Series B: Biological Sciences, 270(suppl 2), S186-S188.

MacIntosh, A. J., Jacobs, A., Garcia, C., Shimizu, K., Mouri, K., Huffman, M. A., \& Hernandez, A. D. (2012). Monkeys in the middle: Parasite transmission through the social network of a wild primate. PloS One, 7(12), e51144.

Makecha, R., Fad, O., \& Kuczaj II, S. A. (2012). The role of touch in the social interactions of Asian elephants (Elephas maximus). International Journal of Comparative Psychology, 25(1), 60-82.

Manno, T. G. (2008). Social networking in the Columbian ground squirrel, Spermophilus columbianus. Animal Behaviour, 75(4), 1221-1228.

McComb, K., Moss, C., Durant, S. M., Baker, L., \& Sayialel, S. (2001). Matriarchs as repositories of social knowledge in African elephants. Science, 292(5516), 491-494.

Nandini, S., Keerthipriya, P., \& Vidya, T. N. C. (2017). Seasonal variation in female Asian elephant social structure in Nagarahole-Bandipur, southern India. Animal Behaviour, 134, 135-145.

Nandini, S., Keerthipriya, P., \& Vidya, T. N. C. (2018). Group size differences may mask underlying similarities in social structure: A comparison of female elephant societies. Behavioral Ecology, 29(1), 145-159.

Newman, M. E. (2004a). Analysis of weighted networks. Physical Review E, 70(5), 056131.

Newman, M. E. (2004b). Fast algorithm for detecting community structure in networks. Physical Review E, 69(6), 066133.

Newman, M. E. (2006). Modularity and community structure in networks. Proceedings of the National Academy of Sciences, 103(23), 8577-8582.

Olson, D. (2004). Elephant husbandry resource guide. International Elephant Foundation.

Plotnik, J. M., \& de Waal, F. B. (2014). Asian elephants (Elephas maximus) reassure others in distress. PeerJ, 2 , e278.

Polthanee, A., \& Promkhambut, A. (2014). Impact of climate change on rice-based cropping systems and farmers' adaptation strategies in Northeast Thailand. Asian Journal of Crop Science, 6(3), 262-272.

Rapaport, L., \& Haight, J. (1987). Some observations regarding allomaternal caretaking among captive Asian elephants (Elephas maximus). Journal of Mammalogy, 68(2), 438-442.

R Core Team (2018). R: A language and environment for statistical computing (version 3.5.1) [Computer software]. R Foundation for Statistical Computing. https://www.R-project.org/.

Revelle, W., \& Revelle, M. W. (2015). Package 'psych'. The Comprehensive R Archive Network. https://cran.rproject.org/web/ packages/psych/psych.pdf.

Rees, P. A. (2009). The sizes of elephant groups in zoos: Implications for elephant welfare. Journal of Applied Animal Welfare Science, 12(1), 44-60.

Rushmore, J., Caillaud, D., Matamba, L., Stumpf, R. M., Borgatti, S. P., \& Altizer, S. (2013). Social network analysis of wild chimpanzees provides insights for predicting infectious disease risk. Journal of Animal Ecology, $82(5), 976-986$.

Schino, G. (2001). Grooming, competition and social rank among female primates: A meta-analysis. Animal Behaviour, 62(2), 265-271. 
Shrout, P. E., \& Fleiss, J. L. (1979). Intraclass correlations: Uses in assessing rater reliability. Psychological Bulletin, $86(2), 420$.

Silk, J., Cheney, D., \& Seyfarth, R. (2013). A practical guide to the study of social relationships. Evolutionary Anthropology: Issues, News, and Reviews, 22(5), 213-225.

Spiess, A. N. (2018). Package 'propagate'. https://CRAN.R-project.org/package=propagate.

Sukumar, R. (1992). The Asian elephant: Ecology and management. Cambridge University Press.

Sukumar, R. (2011). The story of Asia's elephants. Marg Foundation.

Thitaram, C. (2012). Breeding management of captive Asian elephant (Elephas maximus) in range countries and zoos. Japanese Journal of Zoo and Wildlife Medicine, 17(3), 91-96.

van Leeuwen, E. J., Cronin, K. A., Schütte, S., Call, J., \& Haun, D. B. (2013). Chimpanzees (Pan troglodytes) flexibly adjust their behaviour in order to maximize payoffs, not to conform to majorities. PLoS One, 8(11), e80945.

Vidya, T. N. C. (2014). Novel behaviour shown by an Asian elephant in the context of allomothering. Acta Ethologica, $17(2), 123-127$.

Vidya, T. N. C., \& Sukumar, R. (2005). Social organization of the Asian elephant (Elephas maximus) in southern India inferred from microsatellite DNA. Journal of Ethology, 23(2), 205-210.

Williams, R., \& Lusseau, D. (2006). A killer whale social network is vulnerable to targeted removals. Biology Letters, 2(4), 497-500.

Wittemyer, G., \& Getz, W. M. (2007). Hierarchical dominance structure and social organization in African elephants, Loxodonta africana. Animal Behaviour, 73(4), 671-681.

Whitehead, H. (2009). SOCPROG programs: Analysing animal social structures. Behavioral Ecology and Sociobiology, 63(5), 765-778.

Yasui, S., \& Idani, G. I. (2017). Social significance of trunk use in captive Asian elephants. Ethology Ecology \& Evolution, 29(4), 330-350. 


\section{Appendices}

Table A1

Profiles of the Focal Animals

Kaem sean (KS)

Her mahout started to take care of her when she was 10 years old. They experienced street begging in Bangkok and other provinces. Her mahout came back to Ban Ta Klang when he learned she was pregnant. She joined the project in September 2010.

Fah sai (FS)

She was born at her mahout's house. She travelled around Thailand with her mahout to sell jewelry for several years. After that, she worked at an elephant camp. They returned to Ban Ta Klang in 2005 and joined the Surin Project when it started in Mem (ME)

2009. She has had pregnancies and miscarriages, but never given birth to an offspring.

Her mahout started to take care of her when she came to Ban Ta Klang in 2009. She joined the project in 2009 and gave birth to a female in 2010. She was separated from her daughter in February 2012 when her daughter was transferred to another elephant camp in Thailand.

Euang loaung (EL)

Her mahout's family has cared for her throughout her life. She used to have a good relationship with a neonate when she was young. She stayed at an elephant camp, performing street begging. She returned to Ban Ta Klang and joined the Surin Project in January 2012. She has not given birth yet.

Sai faa $(\mathrm{SF})$

Her mahout has worked with her since she was 2.5 years old. She experienced street begging and performed at shows in elephant camps. She joined the project in April 2011. She has never been pregnant.

Kanoon $(\mathrm{KN})$

Her present mahout's family has cared for her since she was young. She spent time together with a neonate when she was young. She was at an elephant camp before she came back to Ban Ta Klang. She joined the project in July 2012. She has not given birth yet.

Gem (GE)

Her present mahout's family has cared for her since 2007. She spent several years street begging. She came to Ban Ta

Klang in 2011 and joined the project in March 2012. She has not given birth yet.

Nong nung (NO)

Her present mahout has cared for her since she was two years old. She was begging on the streets for several years before returning to Ban Ta Klang. She joined the project in January 2011. She has not given birth yet.

Nung ning (NU)

Her mahout started to take care of her when she was three years old. They worked at shows in an elephant camp. They returned to Ban Ta Klang in 2005 and joined the project from the start in 2009. She has not given birth yet.

Teng mo (TM)

She was born at her mahout's home. She spent several years begging on the streets and returned to Ban Ta Klang with her mahout in 2009. She joined the project around May 2011.

Ploy (PL) \& Khwan (KW)

Ploy's mahout started to take care of her when she was six years old. She also experienced street begging in Bangkok and other provinces. After that, she worked as an elephant taxi in an elephant camp. When she got pregnant, her mahout decided to come back to Ban Ta Klang to let her give birth. Khwan was born in 2011. They joined the project in October 2012. 


\section{Table A2}

Results of the Regression Analysis on the Centrality Measures

\begin{tabular}{ccccc}
\hline & Coefficient & SE & $t$ & P (permuted) \\
\hline Degree & & & & \\
& & & & \\
Intercept & 14.696 & 2.512 & 5.850 & 0.323 \\
Age & -0.173 & 0.166 & -1.043 & 0.412 \\
Years in the project & -0.793 & 0.933 & -0.850 & NA \\
Eigenvector Centrality & & & 0.496 \\
Intercept & 0.348 & 0.148 & 2.352 & 0.742 \\
Age & -0.007 & 0.010 & -0.690 & -0.363 \\
Years in the project & -0.020 & 0.055 & & NA \\
Betweenness Centrality & & & 2.188 & 0.586 \\
Intercept & 0.330 & 0.151 & -0.588 & 0.030 \\
Age & -0.006 & 0.010 & 2.700 &
\end{tabular}

Note. $\mathrm{P}$ (permuted) is calculated based on the permutation. 